TRANSACTIONS OF THE

AMERICAN MATHEMATICAL SOCIETY

Volume 358, Number 5, Pages 2105-2122

S 0002-9947(05)03763-3

Article electronically published on May 9, 2005

\title{
INSTABILITY OF STANDING WAVES OF THE SCHRÖDINGER EQUATION WITH INHOMOGENEOUS NONLINEARITY
}

\author{
YUE LIU, XIAO-PING WANG, AND KE WANG
}

\begin{abstract}
This paper is concerned with the inhomogeneous nonlinear Shrödinger equation (INLS-equation)

$$
i u_{t}+\Delta u+V(\epsilon x)|u|^{p} u=0, x \in \mathbf{R}^{N} .
$$

In the critical and supercritical cases $p \geq 4 / N$, with $N \geq 2$, it is shown here that standing-wave solutions of (INLS-equation) on $H^{1}\left(\mathbf{R}^{N}\right)$ perturbation are nonlinearly unstable or unstable by blow-up under certain conditions on the potential term $\mathrm{V}$ with a small $\epsilon>0$.
\end{abstract}

\section{INTRODUCTION}

The nonlinear Schrödinger equation (NLS-equation henceforth)

$$
i u_{t}+\Delta u+|u|^{p} u=0, \quad x \in \mathbf{R}^{N}, t>0,
$$

arises in various physical contexts in the description of a nonlinear wave such as propagation of a laser beam, water waves at the free surface of an ideal fluid and plasma waves. In particular, it models the propagation of intense laser beams in a homogeneous bulk medium with a Kerr nonlinearity. It was suggested that stable high power propagation can be achieved in plasma by sending a preliminary laser beam that creates a channel with a reduced electron density, and thus reduces the nonlinearity inside the channel [5]. Under these conditions, beam propagation can be modeled, in the simplest case, by the following inhomogeneous nonlinear Schödinger equation (INLS-equation in the sequel) of the form

$$
i u_{t}+\Delta u+V(\epsilon x)|u|^{p} u=0, \quad x \in \mathbf{R}^{N}, t>0,
$$

where $N \geq 2$ for $0<p<\frac{4}{N-2}(N \geq 3)$ and $0<p<\infty(N=2)$. The solution $u$ is the electric field in laser and optics, and $V(\epsilon x)$ is proportional to the electron density with a small parameter $\epsilon>0$.

The NLS-equation has been studied by many authors. It is easy to show the local existence of (1.1) [2, 6, 9] in $H^{1}\left(\mathbf{R}^{N}\right)$. That is, there exists $T>0$, such that (1.1) has a unique solution $u \in C\left([0, T), H^{1}\left(\mathbf{R}^{N}\right)\right)$ with the initial data $u_{0} \in H^{1}$,

Received by the editors April 16, 2003 and, in revised form, April 29, 2004.

2000 Mathematics Subject Classification. Primary 35B35, 35B60, 35Q35, 35Q40, 35Q55, 76B25, 76E25, 76E30, 78A15.

Key words and phrases. Nonlinear Schrödinger equation, inhomogeneous nonlinearities, blowup, standing waves, ground state, stability theory.

(C)2005 American Mathematical Society 
and such a $T$ satisfies either $T=\infty$, or

$$
T<+\infty \quad \text { and } \quad \lim _{t \rightarrow T^{-}}\|u(t)\|_{1}=+\infty,
$$

where $\|\cdot\|_{1}$ is the norm in $H^{1}\left(\mathbf{R}^{N}\right)$.

Existence and nonexistence of blow-up solutions of the INLS-equation have been studied by Merle for certain types of inhomogeneities [11. In particular, for the critical power $p=4 / N$, it is shown in 11] that the solution of (1.2) is globally well posed in $H^{1}$ with the $L_{2}$-norm of the initial data $u_{0}$ bounded by $\left|\varphi_{\omega}\right|_{2} / V^{N / 4}(0)$, where $\varphi_{\omega}$ is the unique radially symmetric solution of

$$
\Delta \varphi_{\omega}-\omega \varphi_{\omega}+\varphi_{\omega}^{1+4 / N}=0,
$$

where $|\cdot|_{2}$ is the norm of $L_{2}\left(\mathbf{R}^{N}\right)$. On the other hand, under certain conditions on $V$, Merle [11] showed the existence and lower $L_{2}$-bound of blow-up solutions. Stability of standing waves in the critical case was studied by Fibich and Wang in [4. Their results indicated that stability of the standing waves depends on how its $L_{2}$-norm compares with $V^{N / 2}(0)|R|_{2}^{2}$, where $R$ is the ground state solution of (1.7).

In this paper, attention is given principally to the instability and instability by blow-up of solutions of the INLS-equation. Suppose $V$ satisfies the following properties:

$$
V \text { is radially symmetric, } V(\epsilon x)=V(\epsilon r) \geq V_{0}>0, \quad r=|x|, \epsilon>0,
$$

$\left(H_{0}\right) \quad V \in C^{4} \cap L_{\infty}\left(\mathbf{R}^{N}\right), \quad$ and

$$
\left|V^{(i)}(r)\right| \leq c_{0} e^{r}, \quad i=1,2,3,4,
$$

where $c_{0}$ is a constant and $V^{(i)}$ is the $i$ th derivative of $V$.

It is easy to prove [15, as in the homogeneous case $V=V(0)(2,6], 9])$, that the local existence and uniqueness of the solution of $(1.2) u \in C\left([0, T), H^{1}\right)$ with the initial data $u_{0} \in H^{1}$, and such a time of existence $T$, satisfies either $T=\infty$ or $T<\infty$ and $\lim _{t \rightarrow T^{-}}\|u(t)\|_{1}=\infty$.

Notation. As above and henceforth, we denote the norm of $L_{p}\left(\mathbf{R}^{N}\right)$ by $|\cdot|_{p}$ and the inner product of $L_{2}\left(\mathbf{R}^{N}\right)$ by $(\cdot, \cdot)$. We denote by $\|\cdot\|_{s}$ the norm of Sobolev space $H^{s}\left(\mathbf{R}^{N}\right)$. Here we employ the standard notation $H_{r}^{s}=\left\{u \in H^{s}, \quad u(x)=u(r), r=\right.$ $|x|\}$ with the norm $\|u\|_{s}$. We also denote the integral $\int_{\mathbf{R}^{N}} d x$ simply by $\int$.

By a standing wave, we mean a solution of (1.2) in the form $\psi(x, t)=e^{i \omega t} \varphi_{\omega}(r)$, where $\omega>0$ and $\varphi_{\omega} \in H_{r}^{1}\left(\mathbf{R}^{N}\right)$. It readily follows that $\varphi_{\omega}$ must satisfy the elliptic equation

$$
\Delta \varphi_{\omega}-\omega \varphi_{\omega}+V(\epsilon r) \varphi_{\omega}^{p+1}=0,
$$

where $\varphi_{\omega}^{\prime}(0)=0$, and $\varphi_{\omega}(+\infty)=0$.

The existence of positive solution $\varphi_{\omega} \in H^{1}$ which is called "ground state" has already been proved by Wang and Zeng [16] and Fibich and Wang [4].

Proposition 1.1. Assume $V$ satisfies the condition $\left(H_{0}\right)$. Let $\omega>0$. Then there exists $\epsilon_{0}>0$ and a unique positive solution $\varphi_{\omega}$ in $H^{1}\left(\mathbf{R}^{N}\right)$ to (1.3) for $\forall \epsilon<\epsilon_{0}$. Moreover, $\varphi_{\omega}(r) \leq c_{0} e^{-r / \sqrt{2}}, \forall \epsilon<\epsilon_{0}$ with some constant $c_{0}$.

Proof. See Fibich and Wang [4. 
Equation (1.2) can be written in Hamiltonian form and has the invariants

$$
E(u)=\int \frac{1}{2}|\nabla u|^{2}-\frac{1}{p+2} V(\varepsilon r)|u|^{p+2}
$$

and

$$
Q(u)=\frac{1}{2} \int|u|^{2} .
$$

Stability and instability of standing waves for NLS have been studied by many people [1, 3, 8, 12, 13, 14]. The natural definition of stability of standing waves is nonlinear stability (orbital).

Definition 1.2. The standing wave $\psi(x, t)=e^{i \omega t} \varphi_{\omega}(|x|)$ is nonlinearly stable if for every $\epsilon>0$ there is a $\delta>0$ such that $\inf _{\theta \in \mathbf{R}}\left\|u_{0}-e^{i \theta} \varphi_{\omega}\right\|_{1}<\delta$. Then (1.2) has a unique solution $u \in C\left([0, \infty) ; H^{1}\left(\mathbf{R}^{N}\right)\right)$ with $u(0)=u_{0} \in H^{1}\left(\mathbf{R}^{N}\right)$, and conserved $E(u(t))=E\left(u_{0}\right)$ and $Q(u(t))=Q\left(u_{0}\right) \forall t \geq 0$ such that

$$
\inf _{\theta \in \mathbf{R}}\left\|u(t)-e^{i \theta} \varphi_{\omega}\right\|_{1}<\epsilon
$$

for $0 \leq t<\infty$. Otherwise $\psi=e^{i \omega t} \varphi_{\omega}$ is called nonlinearly unstable.

It is interesting to compare the INLS-equation with the NLS-equation for instability results at the critical case $p=4 / N$. It is known that, at $p=4 / N$, the standing waves of the NLS-equation are strongly unstable 1, 14, i.e. the standing waves are unstable by blow-up in finite time. However, the stability or instability of standing waves for the INLS-equation really depends on the potential term $V$ in the nonlinearity.

In the critical case, $p=4 / N$, Fibich and Wang 4 obtained the stability result of the standing wave $\psi(r, t)=e^{i \omega t} \varphi_{\omega}$ by using the variational techniques [10, 12.

Proposition $1.3([4])$. Let $p=4 / N$, and $\omega>0$. If $V$ satisfies the assumption $\left(H_{0}\right)$ and

$$
V(0) V^{(4)}(0)<G_{N}\left[V^{\prime \prime}(0)\right]^{2}
$$

then $\psi(x, t)=e^{i \omega t} \varphi_{\omega}(r)$ is nonlinearly stable in $H^{1}\left(\mathbf{R}^{N}\right)$ for $\epsilon$ small enough, where

$$
G_{N}=\frac{6(N+2)}{N} \frac{\int_{\mathbf{R}^{N}} r^{2} R^{\frac{4}{N}+1} L_{0}^{-1}\left(r^{2} R^{\frac{4}{N}+1}\right)}{\int_{\mathbf{R}^{N}} r^{4} R^{2+4 / N}},
$$

$R(r)$ is the ground state of

$$
\Delta R-R+R^{1+4 / N}=0
$$

and $L_{0}=\Delta-1+\left(1+\frac{4}{N}\right) R^{4 / N}$.

Remark. It was also shown in [4] numerically that $G_{N}<0$ when $N=2$. In fact, we prove in Section 3 that $G_{N}<0$ for all $N \geq 2$. In this case, $V^{(4)}(0)$ is necessarily negative for stability of the standing waves.

Define a function $d(\omega)$ which plays a central role in stability and instability by

$$
d(\omega)=E\left(\varphi_{\omega}\right)+\omega Q\left(\varphi_{\omega}\right) .
$$

It is easy to see that the stability condition (1.6) is equivalent to the convexity of $d$, that is, $d^{\prime \prime}(\omega)>0$. Notice that for the regular NLS, $d^{\prime \prime}(\omega)=0$ in the critical case.

It is our purpose here to show instability of standing waves (Theorem 2.3) and strong instability (Theorem 2.5) for INLS or (1.2) in critical or supercritical cases. 
The results complement those of Fibich and Wang 4 which dealt with the stability only in the critical case. In particular, our results show that in the critical case, a sufficient condition for instability is when $V^{(4)}(0)>0$. It is not known what happens when $V^{(4)}(0)$ is negative but (1.6) is violated.

To establish the instability results in view, we are basically following the argument of Goncalves Rebeio [7] who studied the case of the nonlinear Schrödinger equation with external magnetic field. It is noted that INLS is different from NLS with the pure power nonlinearity, scaling and dilation technique does not give the description of action $d(\omega)$ explicitly. Thus, we cannot apply the Grillakis-ShatahStrauss abstract formalism [8] or the Shatah-Strauss [1] techniques for instability. Using detailed analysis with variational characterization, we are able to construct unstable flow near the standing wave.

The plan of the paper is as follows. In Section 2, some variational properties of standing waves and the principal results of instability (Theorem 2.3) and strong instability (Theorem 2.5) are described. Section 3 is devoted to the result of instability of standing waves and the technical development. Then the instability of standing waves by blow up in finite time is established in Section 4 .

\section{The StAnding WAVES AND The Instability RESUlts}

We define functionals $L, I$ and $P$ in the following:

$$
\begin{gathered}
L(u)=E(u)+\omega Q(u)=\frac{1}{2} \int|\nabla u|^{2}-\frac{1}{p+2} \int V(\varepsilon r)|u|^{p+2}+\frac{\omega}{2} \int|u|^{2}, \\
I(u)=\int|\nabla u|^{2}-\int V(\varepsilon r)|u|^{p+2}+\omega \int|u|^{2} \text { and } \\
P(u)=\int|\nabla u|^{2}-\frac{1}{p+2} \int\left(\frac{p N}{2} V(\varepsilon r)-\varepsilon r V^{\prime}(\varepsilon r)\right)|u|^{p+2} .
\end{gathered}
$$

Let $u^{\lambda}(x)=\lambda^{\frac{N}{2}} u(\lambda x)$. A simple computation shows that

$$
\frac{d}{d \lambda} L\left(u^{\lambda}\right)=\frac{1}{\lambda} P\left(u^{\lambda}\right)
$$

On the other hand, the following virial identity can be easily verified [11]:

$$
\frac{d^{2}}{d t^{2}} \int r^{2}|u(r, t)|^{2}=8 P(u)
$$

where $u$ is the solution of INLS with the initial data in the space

$$
\Sigma=\left\{u \in H_{r}^{1}\left(\mathbf{R}^{N}\right), \quad r u \in L_{2}\left(\mathbf{R}^{N}\right)\right\} .
$$

The proof of (2.5) for classical solutions is given by Glassey (referenced in [1]).

Lemma 2.1. If $\varphi_{\omega}$ is a positive solution of (1.3), then $L\left(\varphi_{\omega}\right)=\inf \{L(u), u \neq$ $\left.0, L_{\omega}^{\prime}(u)=0\right\}$ and the following Pohozaev identities hold, i.e. $P\left(\varphi_{\omega}\right)=0$ and $I_{\omega}\left(\varphi_{\omega}\right)=0$.

Proof. The proof of the first part of the lemma can be found in [4]. For the Pohozaev identities, define $\varphi_{\omega}^{\lambda}=\lambda^{N / 2} \varphi_{\omega}(\lambda r)$ with $\lambda>0$. Then one obtains that

$$
P\left(\varphi_{\omega}\right)=\left.\frac{d}{d \lambda} L\left(\varphi_{\omega}^{\lambda}\right)\right|_{\lambda=1}=\left\langle L^{\prime}\left(\varphi_{\omega}\right),\left.\frac{d \varphi_{\omega}^{\lambda}}{d \lambda}\right|_{\lambda=1}\right\rangle=0 .
$$

Similarly, we can prove $I\left(\varphi_{\omega}\right)=0$ by $\phi_{\omega}^{\lambda}(x)=\lambda \varphi_{\omega}(r)$. 


\section{Lemma 2.2.}

$$
d(\omega)=\inf \left\{L(u) ; \quad u \neq 0, u \in H_{r}^{1}\left(\mathbf{R}^{N}\right), I(u)=0\right\},
$$

where $L(u)$ and $I(u)$ are defined in (2.1) and (2.2), respectively.

Recall $d(\omega)=E\left(\varphi_{\omega}\right)+\omega Q\left(\varphi_{\omega}\right)=L\left(\varphi_{\omega}\right)$ defined in (1.8).

Proof. Define

$$
d_{0}=\inf \left\{L(u) ; \quad u \neq 0, u \in H_{r}^{1}\left(\mathbf{R}^{N}\right), I(u)=0\right\} .
$$

First it is observed that there is some $u \in H_{r}^{1}\left(\mathbf{R}^{N}\right)$ such that $I(u)=0$. Indeed, if $I(u)<0$, , then we set $u^{\lambda}=\lambda^{N / 2} u(\lambda r)$ and it follows that

$$
I\left(u^{\lambda}\right)=\omega \int|u|^{2}+\lambda^{2} \int|\nabla u|^{2}-\lambda^{\frac{p N}{2}} \int V\left(\frac{\epsilon r}{\lambda}\right)|u|^{p+2} .
$$

Since $I\left(u^{\lambda}\right)>0$, as $\lambda \rightarrow 0$ and $I\left(u^{1}\right)=I(u)<0$, there exists some $\lambda_{0} \in(0,1)$ such that $I\left(u^{\lambda_{0}}\right)=0$ by the continuity of $I$ at $\lambda$. Next suppose $\left\{u_{n}\right\} \in H_{r}^{1}$ is a minimizing sequence of $(2.7)$ with $I\left(u_{n}\right)=0$ and $\lim _{n \rightarrow \infty} L\left(u_{n}\right)=d(\omega)$. We can assume that $u_{n} \geq 0$. Otherwise we can consider $u_{n}^{+}=\underset{n \rightarrow \infty}{=} \max \left\{u_{n}, 0\right\} \geq 0$. It is clear that the problem (2.7) is equivalent to

$$
d(\omega)=\inf \left\{\left(\frac{1}{2}-\frac{1}{p+2}\right) \int|\nabla u|^{2}+\omega|u|^{2} ; \quad u \neq 0, \quad u \in H_{r}^{1}\left(\mathbf{R}^{N}\right), I(u)=0\right\} .
$$

It follows from (2.9) that $u_{n}$ is bounded in $H_{r}^{1}\left(\mathbf{R}^{N}\right)$. Therefore there exists a subsequence, denoted again by $u_{n}$, such that $u_{n} \rightarrow u_{0} \in H_{r}^{1}\left(\mathbf{R}^{N}\right)$ weakly. By the following compact embedding (Strauss' Lemma): $H_{r}^{1}\left(\mathbf{R}^{N}\right) \hookrightarrow L_{p+2}\left(\mathbf{R}^{N}\right)$ for $N \geq 2$, we get $u_{n} \rightarrow u_{0}$ strongly in $L_{p+2}\left(\mathbf{R}^{N}\right)$ after choosing some subsequence.

Now we claim $u_{0} \neq 0$. In fact, by Gagliardo-Nirenberg inequality and $I\left(u_{n}\right)=0$,

$$
\begin{aligned}
\left|u_{n}\right|_{p+2}^{2} & \leq C_{p, N}\left|\nabla u_{n}\right|_{2}^{\frac{p N}{p+2}}\left|u_{n}\right|_{2}^{\frac{4-p(N-2)}{p+2}} \leq C_{p, N, \omega}\left(\left|\nabla u_{n}\right|_{2}^{2}+w\left|u_{n}\right|_{2}^{2}\right) \\
& \leq C_{p, N, \omega}\left|u_{n}\right|_{p+2}^{p+2} .
\end{aligned}
$$

This implies $\left|u_{n}\right|_{p+2} \geq C_{p, N, \omega}>0$. Therefore it follows from the strong limit $u_{n} \rightarrow u_{0}$ in $L_{p+2}\left(\mathbf{R}^{N}\right)$ that $u_{0} \neq 0$. By the lower-semicontinuity, one obtains

$$
\begin{aligned}
d_{0} & =\lim _{n \rightarrow \infty}\left(\frac{1}{2}-\frac{1}{p+2}\right) \int\left|\nabla u_{n}\right|^{2}+\omega\left|u_{n}\right|^{2} \\
& \geq\left(\frac{1}{2}-\frac{1}{p+2}\right) \int\left|\nabla u_{0}\right|^{2}+\omega\left|u_{0}\right|^{2}
\end{aligned}
$$

and

$$
I\left(u_{0}\right) \leq \lim _{n \rightarrow \infty} \int\left|\nabla u_{n}\right|^{2}+w\left|u_{n}\right|^{2}-V(\epsilon r)\left|u_{n}\right|^{2}=0 .
$$

If $I\left(u_{0}\right)<0$, we choose $u_{0}^{\lambda}=\lambda^{N / 2} u_{0}(\lambda r)$ with $\lambda \in(0,1)$ such that $I\left(u_{0}^{\lambda}\right)=0$. It follows from (2.10) that

$$
\begin{aligned}
L\left(u_{0}^{\lambda}\right) & =\left(\frac{1}{2}-\frac{1}{p+2}\right) \int \lambda^{2}\left|\nabla u_{0}\right|^{2}+\omega\left|u_{0}\right|^{2} \\
& <\left(\frac{1}{2}-\frac{1}{p+2}\right) \int\left|\nabla u_{0}\right|^{2}+\omega\left|u_{0}\right|^{2} \leq d_{0}
\end{aligned}
$$


which contradicts the definition of $d_{0}$. Consequently we have $I\left(u_{0}\right)=0$ and $L\left(u_{0}\right)=$ $d_{0}$.

By Lagrange-Euler equation, there exists a Lagrange multiplier $\theta$ such that

$$
L^{\prime}\left(u_{0}\right)+\theta I^{\prime}\left(u_{0}\right)=0
$$

and

$$
\left\langle L^{\prime}\left(u_{0}\right), u_{0}\right\rangle+\theta\left\langle I^{\prime}\left(u_{0}\right), u_{0}\right\rangle=0 .
$$

Since $I\left(u_{0}\right)=\left\langle L^{\prime}\left(u_{0}\right), u_{0}\right\rangle=0$, it follows from (2.13) that

$$
\theta \int V(\epsilon r)\left|u_{0}\right|^{p+2}=0
$$

It is concluded that $\theta=0$ because $u_{0} \neq 0$. Therefore $u_{0}$ solves the equation $L^{\prime}\left(u_{0}\right)=$ 0 , that is, $u_{0}$ is the positive solution of (1.3) in $H_{r}^{1}\left(\mathbf{R}^{N}\right)$. By the uniqueness of the solution of (1.3) in $H_{r}^{1}\left(\mathbf{R}^{N}\right), u_{0}=\varphi_{\omega}$ and $d_{0}=d(\omega)$. This completes the proof of Lemma 2.2 .

One of the main results of the present paper is the nonlinear instability of standing waves in $H^{1}\left(\mathbf{R}^{N}\right)$. It is stated as follows.

Theorem 2.3 (Instability). Assume $\left(H_{0}\right)$ holds. Let $w>0$ and let $\varphi_{\omega}$ be the ground-state solution of (1.3). If $\left.\partial_{\lambda}^{2} E\left(\varphi_{\omega}^{\lambda}\right)\right|_{\lambda=1}<0$, then $\psi(r, t)=e^{i w t} \varphi_{\omega}(r)$ is nonlinearly unstable in $H^{1}$, where $\varphi_{\omega}^{\lambda}(r)=\lambda^{\frac{N}{2}} \varphi_{\omega}(\lambda r), \lambda>0$, and $r=|x|$.

Corollary 2.4. Assume $\left(H_{0}\right)$ holds. Let $\omega>0$. Then there exists a $\varepsilon_{0}>0$ such that for any $0<\varepsilon<\varepsilon_{0},\left.\partial_{\lambda}^{2} E\left(\varphi_{\omega}^{\lambda}\right)\right|_{\lambda=1}<0$, if

(a) $p>4 / N$, or

(b) $p=4 / N$ and $V^{(4)}(0)>0$.

Therefore, in either case, $\psi=e^{i \omega t} \varphi_{\omega}(r)$ is nonlinearly unstable in $H^{1}$.

Furthermore, using the virial identity (2.5) with detailed analysis for invariant sets, we are able to show the following result of strong instability.

Theorem 2.5 (Instability by blow up). Assume $\left(H_{0}\right)$ holds. Let $\omega>0$. If $V$ satisfies the conditions

a) $p=4 / N: V^{(4)}(0)>0$ or

b) $p>4 / N$ : either $V^{\prime \prime}(0)<0$ or $V^{\prime \prime}(0)=0, V^{(4)}(0)>0$ with $p<8 / N$,

then the standing wave $\psi=e^{i \omega t} \varphi_{\omega}(r)$ is nonlinearly unstable in the following sense. For any $\delta>0$, there exists $T<+\infty$ and a function $u_{0} \in H^{1}\left(\mathbf{R}^{N}\right)$ with $\left\|u_{0}-\varphi_{\omega}\right\|_{1}<$ $\delta$, such that the solution $u$ of $(1.2)$ with $u(0)=u_{0}$ satisfies

$$
\lim _{t \rightarrow T}|\nabla u(t)|_{2}=+\infty \text {. }
$$

\section{Nonlinear InStability}

In this section, we give a proof of our main results stated above. The proof is basically following the argument of Goncalves Rebeiro [7], which is a detour of the Grillakis-Shatah-Strauss formalism [8]. If dilation and scaling would yield the $\omega$ variable trajectory, the convexity of concavity separates stability from instability by the action $d(\omega)$. However, in the present case, such trajectory cannot be obtained by scaling and dilation because of the inhomogeneous nonlinearities. 
For $\epsilon_{0}>0$, define a tubular neighborhood around the orbit $\left\{e^{i \theta} \varphi_{\omega} ; \theta \in \mathbf{R}\right\}$ by

$$
U_{\varepsilon_{0}}\left(\varphi_{\omega}\right)=\left\{v \in H_{r}^{1}\left(\mathbf{R}^{N}\right) ; \quad \inf _{\theta \in \mathbf{R}}\left\|v-e^{i \theta} \varphi_{\omega}\right\|_{1}<\varepsilon_{0}\right\} .
$$

The proof of Theorem 2.3 is approached via a series of lemmas.

Lemma 3.1. If $\left.\partial_{\lambda}^{2} E\left(\varphi_{\omega}^{\lambda}\right)\right|_{\lambda=1}<0$, where $\varphi_{\omega}^{\lambda}=\lambda^{\frac{N}{2}} \varphi_{\omega}(\lambda x)$ with $\lambda>0$, then there exist $\varepsilon_{0}>0, \delta_{0}>0$ and a mapping $\lambda: U_{\epsilon_{0}}\left(\varphi_{\omega}\right) \rightarrow\left(1-\delta_{0}, 1+\delta_{0}\right)$ such that

$$
I\left(v^{\lambda(v)}\right)=0 \quad \forall v \in U_{\epsilon_{0}}\left(\varphi_{\omega}\right),
$$

where $v^{\lambda}=\lambda^{\frac{N}{2}} v(\lambda x)$ and $I(u)$ is defined in $(2.2)$ i.e.

$$
I(u)=\int|\nabla u|^{2}-\int V(\varepsilon r)|u|^{p+2}+\omega \int|u|^{2} .
$$

Proof. A simple calculation shows that

$$
\left.\frac{\partial}{\partial \lambda} I\left(v^{\lambda}\right)\right|_{\lambda=1, v=\varphi_{\omega}}=\left\langle I^{\prime}\left(\varphi_{\omega}\right),\left.\frac{\partial \varphi_{\omega}^{\lambda}}{\partial \lambda}\right|_{\lambda=1}\right\rangle=\left\langle I_{\omega}^{\prime}\left(\varphi_{\omega}\right), \Phi_{\omega}\right\rangle
$$

where $\Phi_{\omega}=\frac{\partial \varphi_{\omega}^{\lambda}}{\partial \lambda}$

Note that $\left\langle I^{\prime}\left(\varphi_{\omega}\right), \Phi_{\omega}\right\rangle \neq 0$. Indeed, if $\left\langle I_{\omega}^{\prime}\left(\varphi_{\omega}\right), \Phi_{\omega}\right\rangle=0$, then $\Phi_{\omega}$ would be tangent to $S$ at $\varphi_{\omega}$, where

$$
S=\left\{u \in H_{r}^{1}\left(\mathbf{R}^{N}\right) ; \quad u \neq 0, I(u)=0\right\} .
$$

In this case, $\left\langle L_{\omega}^{\prime \prime}\left(\varphi_{\omega}\right) \Phi_{\omega}, \Phi_{\omega}\right\rangle \geq 0$ since $\varphi_{\omega}$ minimizes $L(u)$ on $S$ by Lemma 2.2 . This leads to a contradiction of the assumption

$$
\left.\partial_{\lambda}^{2} E\left(\varphi_{\omega}^{\lambda}\right)\right|_{\lambda=1}=\left\langle L^{\prime \prime}\left(\varphi_{\omega}\right) \Phi_{\omega}, \Phi_{\omega}\right\rangle<0
$$

where $\left.\partial_{\lambda} E\left(\varphi_{\omega}^{\lambda}\right)\right|_{\lambda=1}=\left\langle L^{\prime}\left(\varphi_{\omega}\right), \Phi_{\omega}\right\rangle=0$.

The result is then obtained by the implicit function theorem with

$$
\left.I\left(v^{\lambda}\right)\right|_{\substack{\lambda=1 \\ v=\varphi_{\omega}}}=I\left(\varphi_{\omega}\right)=0 .
$$

This completes the proof of Lemma 3.1.

Lemma 3.2. If $\left.\partial_{\lambda}^{2} E\left(\varphi_{\omega}^{\lambda}\right)\right|_{\lambda=1}<0$, then there exist $\varepsilon_{1}>0$ and $\delta_{1}>0$ with the following property: for any $v \in U_{\varepsilon_{1}}\left(\varphi_{\omega}\right)$ satisfying $|v|_{2}=\left|\varphi_{\omega}\right|_{2}$, there exists $\lambda(v) \in$ $\left(1-\delta_{1}, 1+\delta_{1}\right)$ such that

$$
E\left(\varphi_{\omega}\right)<E(v)+(\lambda(v)-1) P(v),
$$

where $P(v)$ is defined in (2.3).

Proof. From the assumption $\left.\partial_{\lambda}^{2} E\left(\varphi_{\omega}^{\lambda}\right)\right|_{\lambda=1}<0$ and the continuity of $\partial_{\lambda}^{2} E\left(v^{\lambda}\right)$ in $\lambda$ and $v$, there exist $\epsilon_{1}>0$ and $\delta_{1}>0$ such that $\partial_{\lambda}^{2} E\left(v^{\lambda}\right)<0$ for all $\lambda \in\left(1-\delta_{1}, 1+\delta_{1}\right)$ and $v \in U_{\epsilon_{1}}\left(\varphi_{\omega}\right)$. Since $\left.\partial_{\lambda} E\left(v^{\lambda}\right)\right|_{\lambda=1}=\left.\frac{1}{\lambda} P\left(v^{\lambda}\right)\right|_{\lambda=1}=P(v)$, the Taylor expansion at $\lambda=1$ gives

$$
E\left(v^{\lambda}\right)<E(v)+(\lambda-1) P(v)
$$

for $\lambda \in\left(1-\delta_{1}, 1+\delta_{1}\right)$ and $v \in U_{\varepsilon_{1}}\left(\varphi_{\omega}\right)$.

By Lemma 3.1, we can take $\delta_{1}>0, \varepsilon_{1}>0$ such that $\delta_{1}<\delta_{0}, \varepsilon_{1}<\varepsilon_{0}$ small enough so that there exists $\lambda(v) \in\left(1-\delta_{1}, 1+\delta_{1}\right)$, and $I\left(v^{\lambda(v)}\right)=0$ for any 
$v \in U_{\varepsilon_{1}}\left(\varphi_{\omega}\right)$. Furthermore, from Lemma 2.2 if $|v|_{2}=\left|\varphi_{\omega}\right|_{2}$ such that $I\left(v^{\lambda(v)}\right)=0$, then one obtains from the fact that $Q\left(v^{\lambda}\right)=Q(v)$ that

$$
\begin{aligned}
E\left(v^{\lambda(v)}\right) & =L\left(v^{\lambda(v)}\right)-\omega Q\left(v^{\lambda(v)}\right) \geq L\left(\varphi_{\omega}\right)-\omega Q(v) \\
& =L\left(\varphi_{\omega}\right)-\omega Q\left(\varphi_{\omega}\right)=E\left(\varphi_{\omega}\right) .
\end{aligned}
$$

Therefore, from (3.4) and (3.5), we obtain (3.3). This completes the proof of Lemma 3.2 .

We next define

$$
A=\left\{v \in U_{\varepsilon_{1}}\left(\varphi_{\omega}\right), \quad E(v)<E\left(\varphi_{\omega}\right),|v|_{2}=\left|\varphi_{\omega}\right|_{2}, P(v)<0\right\} .
$$

For any $u_{0} \in U_{\varepsilon_{1}}\left(\varphi_{\omega}\right)$, we define the exit time from $U_{\varepsilon_{1}}\left(\varphi_{\omega}\right)$ as follows:

$$
T\left(u_{0}\right)=\sup \left\{T>0 . \quad u(t) \in U_{\varepsilon_{1}}\left(\varphi_{\omega}\right), \quad 0 \leq t<T\right\},
$$

where $u(t)$ is the solution of (1.2).

Lemma 3.3. If $\left.\partial_{\lambda}^{2} E\left(\varphi_{\omega}^{\lambda}\right)\right|_{\lambda=1}<0$, then for any $u_{0} \in A$, there exists $\delta_{0}=\delta_{0}\left(u_{0}\right)>0$ such that $P(u(t))<-\delta_{0}$ for $0 \leq t<T\left(u_{0}\right)$.

Proof. For $u_{0} \in A$, let $\delta_{2}=E\left(\varphi_{\omega}\right)-E\left(u_{0}\right)>0$. From Lemma 3.2 and the conservation laws $E(u(t))=E\left(u_{0}\right)$ and $Q(u(t))=Q\left(u_{0}\right)$, we have

$$
0<\delta_{2}<(\lambda(u(t))-1) P(u(t)), \quad 0 \leq t<T\left(u_{0}\right) .
$$

Thus, $P(u(t)) \neq 0$ for $0 \leq t<T\left(u_{0}\right)$ and $|\lambda(u(t))-1|<\delta_{1}$. Since the mapping $t \longmapsto P(u(t))$ is continuous and $P\left(u_{0}\right)<0$, we have $P(u(t))<0$ by (3.6) for $0 \leq t<T\left(u_{0}\right)$. It follows from (3.6) that

$$
\begin{aligned}
& -\delta_{1}<\lambda(u(t))-1<0, \quad 0 \leq t<T\left(u_{0}\right), \\
& -P(u(t))>\frac{\delta_{2}}{1-\lambda(u(t))} \geq \frac{\delta_{2}}{\delta_{1}}, \quad 0 \leq t<T\left(u_{0}\right) .
\end{aligned}
$$

Taking $\delta_{0}=\frac{\delta_{2}}{\delta_{1}}$, the proof of Lemma 3.3 is completed.

Now we are in a position to prove Theorem 2.3.

Proof of Theorem 2.3. Since $\left.\partial_{\lambda} E\left(\varphi_{\omega}^{\lambda}\right)\right|_{\lambda=1}=0,\left.\partial_{\lambda}^{2} E\left(\varphi_{\omega}^{\lambda}\right)\right|_{\lambda=1}<0$ and $P\left(\varphi_{\omega}^{\lambda}\right)=$ $\lambda \partial_{\lambda} E\left(\varphi_{\omega}^{\lambda}\right)$ with $Q\left(\varphi_{\omega}^{\lambda}\right)=Q\left(\varphi_{\omega}\right)$, we have $E\left(\varphi_{\omega}^{\lambda}\right)<E\left(\varphi_{\omega}\right)$ and $P\left(\varphi_{\omega}^{\lambda}\right)<0$ for $\lambda>1$ sufficiently close to 1 . Moreover, $\left|\varphi_{\omega}^{\lambda}\right|_{2}=\left|\varphi_{\omega}\right|_{2}$ and $\left\|\varphi_{\omega}^{\lambda}-\varphi_{\omega}\right\|_{1} \rightarrow 0$ as $\lambda \rightarrow 1^{+}$, which implies $\varphi_{\omega}^{\lambda} \in A$ for $\lambda \rightarrow 1^{+}$.

By the exponential decay of $\varphi_{\omega}$ in $r$, it is clear that $\int r^{2}\left|\varphi_{\omega}^{\lambda}(r)\right|^{2}<\infty$. Hence it follows from the virial identity $(2.5)$

$$
\frac{d^{2}}{d t^{2}} \int r^{2}\left|u_{\lambda}(t, r)\right|^{2}=8 P\left(u_{\lambda}(t)\right) \quad 0 \leq t<T\left(\varphi_{\omega}^{\lambda}\right),
$$

where $u_{\lambda}(t)$ is the solution of (1.2) with the initial data $u_{\lambda}(0)=\varphi_{\omega}^{\lambda}$. From Lemma 3.3 , there exists $\delta_{\lambda}>0$ such that

$$
P\left(u_{\lambda}(t)\right)<-\delta_{\lambda}, \quad 0 \leq t<T\left(\varphi_{\omega}^{\lambda}\right) .
$$

Hence from (3.7), (3.8) and the inequality $|u|_{2} \leq \frac{2}{N}|\nabla u|_{2}|r u|_{2}$, one can conclude that $T\left(\varphi_{\omega}^{\lambda}\right)<\infty$. The proof of Theorem 2.3 is now completed. 
Proof of Corollary 2.4. We estimate $\partial_{\lambda}^{2} E\left(\varphi_{\omega}^{\lambda}\right)$ at $\lambda=1$ for small $\epsilon>0$ in the following. First we observe from (2.3) that

$$
\begin{gathered}
P\left(\varphi_{\omega}^{\lambda}\right)=\lambda^{2} \int\left|\nabla \varphi_{\omega}\right|^{2}-\frac{\lambda^{\frac{p N}{2}}}{p+2} \int\left(\frac{p N}{2} V\left(\frac{\epsilon r}{\lambda}\right)-V^{\prime}\left(\frac{\epsilon r}{\lambda}\right) \frac{\epsilon r}{\lambda}\right)\left|\varphi_{\omega}\right|^{p+2}, \\
Q\left(\varphi_{\omega}^{\lambda}\right)=Q\left(\varphi_{\omega}\right)=\frac{1}{2} \int\left|\varphi_{\omega}\right|^{2}
\end{gathered}
$$

and

$$
\frac{\partial}{\partial \lambda} E\left(\varphi_{\omega}^{\lambda}\right)=\frac{\partial}{\partial \lambda} L\left(\varphi_{\omega}^{\lambda}\right)=\frac{1}{\lambda} P\left(\varphi_{\omega}^{\lambda}\right)
$$

Hence

$$
\begin{aligned}
\left.\frac{\partial^{2}}{\partial \lambda^{2}} E\left(\varphi_{\omega}^{\lambda}\right)\right|_{\lambda=1} & =\left.\frac{\partial^{2}}{\partial \lambda^{2}} L\left(\varphi_{\omega}^{\lambda}\right)\right|_{\lambda=1}=\left.\left(-\frac{1}{\lambda^{2}} P\left(\varphi_{\omega}^{\lambda}\right)+\frac{1}{\lambda} \frac{\partial}{\partial \lambda} P\left(\varphi_{\omega}^{\lambda}\right)\right)\right|_{\lambda=1} \\
& =\left.\frac{1}{\lambda} \frac{\partial}{\partial \lambda} P\left(\varphi_{\omega}^{\lambda}\right)\right|_{\lambda=1},
\end{aligned}
$$

because $\left.P\left(\varphi_{\omega}^{\lambda}\right)\right|_{\lambda=1}=P\left(\varphi_{\omega}\right)=0$. A direct calculation gives

$$
\begin{aligned}
& \left.\frac{\partial}{\partial \lambda} P\left(\varphi_{\omega}^{\lambda}\right)\right|_{\lambda=1} \\
& =2\left[\int\left|\nabla \varphi_{\omega}\right|^{2}-\frac{p N}{4(p+2)} \int\left(\frac{p N}{2} V(\epsilon r)-V^{\prime}(\epsilon r)(\epsilon r)\right)\left|\varphi_{\omega}\right|^{p+2}\right. \\
& \left.0)-\frac{1}{2(p+2)} \int\left(-\frac{p N}{2} V^{\prime}(\epsilon r) \epsilon r+V^{\prime}(\epsilon r) \epsilon r+V^{\prime \prime}(\epsilon r)(\epsilon r)^{2}\right)\left|\varphi_{\omega}\right|^{p+2}\right] \\
& =2 \int\left|\nabla \varphi_{\omega}\right|^{2}-\frac{1}{p+2} \int\left(\frac{(p N)^{2}}{4} V(\epsilon r)+(1-p N) V^{\prime}(\epsilon r)(\epsilon r)\right. \\
& \left.+V^{\prime \prime}(\epsilon r)(\epsilon r)^{2}\right)\left|\varphi_{\omega}\right|^{p+2}
\end{aligned}
$$

On the other hand, from $P\left(\varphi_{\omega}\right)=0$, one obtains

$$
\left|\nabla \varphi_{\omega}\right|^{2}=\frac{p N}{2(p+2)} \int V(\epsilon r)\left|\varphi_{\omega}\right|^{p+2}-\frac{1}{p+2} \int \epsilon r V^{\prime}(\epsilon r)\left|\varphi_{\omega}\right|^{p+2}
$$

Therefore

$$
\begin{aligned}
& \left.\frac{\partial^{2}}{\partial \lambda^{2}} E\left(\varphi_{\omega}^{\lambda}\right)\right|_{\lambda=1} \\
& =\frac{1}{p+2} \int\left[\left(2-\frac{p N}{2}\right) \frac{p N}{2} V(\varepsilon r)-(3-p N) V^{\prime}(\varepsilon r) \varepsilon r-\varepsilon^{2} r^{2} V^{\prime \prime}(\varepsilon r)\right]\left|\varphi_{\omega}\right|^{p+2}
\end{aligned}
$$


For a small $\epsilon>0$, Taylor expansion shows that

$$
\begin{aligned}
& \left(2-\frac{p N}{2}\right) \frac{p N}{2} V(\varepsilon r)-(3-p N) V^{\prime}(\varepsilon r) \varepsilon r-\varepsilon^{2} r^{2} V^{\prime \prime}(\varepsilon r) \\
& =\left(2-\frac{p N}{2}\right) \frac{p N}{2}\left(V(0)+\frac{1}{2} V^{\prime \prime}(0)(\varepsilon r)^{2}+\frac{1}{4} V^{(4)}(0)(\varepsilon r)^{4}\right) \\
& -(3-p N)\left(V^{\prime \prime}(0)(\varepsilon r)^{2}+\frac{1}{6} V^{(4)}(0)(\varepsilon r)^{4}\right) \\
r & -V^{\prime \prime}(0)(\varepsilon r)^{2}-\frac{1}{2} V^{(4)}(0)(\varepsilon r)^{4}+O\left((\epsilon r)^{6}\right) \\
& =\left(2-\frac{p N}{2}\right) \frac{p N}{2} V(0)+\left[\left(2-\frac{p N}{2}\right) \frac{p N}{4}-(3-p N)-1\right] V^{\prime \prime}(0)(\varepsilon r)^{2} \\
& +\left[\left(2-\frac{p N}{2}\right) \frac{p N}{48}-\frac{1}{6}(3-p N)-\frac{1}{2}\right] V^{(4)}(0)(\varepsilon r)^{4}+O\left((\epsilon r)^{6}\right) \\
& =\left(2-\frac{p N}{2}\right) \frac{p N}{2} V(0)+\frac{1}{2}\left(2-\frac{p N}{2}\right)\left(\frac{p N}{2}-4\right) V^{\prime \prime}(0)(\varepsilon r)^{2} \\
& +\left[\left(2-\frac{p N}{2}\right) \frac{p N}{48}-\frac{1}{3}\left(3-\frac{p N}{2}\right)\right] V^{(4)}(0)(\varepsilon r)^{4}+O\left((\epsilon r)^{6}\right) .
\end{aligned}
$$

By Proposition 1.1, $\int O\left((\epsilon r)^{6}\right)\left|\varphi_{\omega}\right|^{p+2}=O\left(\epsilon^{6}\right)$, as $\epsilon \rightarrow 0$. It follows from (3.12) that $\left.\frac{\partial^{2}}{\partial \lambda^{2}} E\left(\varphi_{\omega}^{\lambda}\right)\right|_{\lambda=1}<0 \Longleftrightarrow \exists \varepsilon_{0}>0$ for any $0<\varepsilon<\varepsilon_{0}$, either

(a) $p>\frac{4}{N}$, or

(b) $\quad p=\frac{4}{N}$ and $V^{(4)}(0)>0$.

The proof of Corollary 2.4 is completed.

Now we show that $G_{N}<0$ for any $N \geq 2$.

Theorem 3.4. Let $p=\frac{4}{N}$ with $N \geq 2$ and

$$
G_{N}=\frac{6(N+2)}{N} \frac{\int r^{2} R^{\frac{4}{N}+1} L_{0}^{-1}\left(r^{2} R^{1+\frac{4}{N}}\right)}{\int r^{4} R^{2+\frac{4}{N}}}
$$

defined in Proposition 1.3, and ground state $R$ is defined in (1.7). Then $G_{N}<0$.

In order to prove Theorem 3.4, we need the following lemma.

Lemma 3.5. $\inf _{(f, R)=0}\left(L_{0} f, f\right)=0$, where $(\cdot, \cdot)$ is the $L_{2}$-inner product.

Proof. See Proposition 2.7 in [13].

Proof of Theorem 3.4. To show that $G_{N}<0$, it suffices to prove

$$
\int r^{2} R^{\frac{4}{N}+1} g>0
$$

where $\left(-L_{0}\right) g=r^{2} R^{\frac{4}{N}+1}$, that is, $g=g(r)$ satisfies the equation

$$
-\triangle g+g-\left(1+\frac{4}{N}\right) R^{\frac{4}{N}} g=r^{2} R^{\frac{4}{N}+1},
$$

or we need to show that

$$
\left(-L_{0} g, g\right)=\int r^{2} R^{\frac{4}{N}+1} g>0 .
$$


According to Lemma 3.5, first, we claim

$$
\int R g=0
$$

so that we can apply Lemma 3.5 to obtain

$$
\int r^{2} R^{\frac{4}{N}+1} g \geq 0
$$

After (3.17) is proved, we will show

$$
\int r^{2} R^{\frac{4}{N}+1} g \neq 0 .
$$

Claim. $\int R g=0$.

In fact, multiplying (1.7) by $g$ and using integration by parts yields

$$
-\int \nabla R \cdot \nabla g-\int R g+\int R^{1+\frac{4}{N}} g=0 .
$$

Similarly, multiplying (3.14) by $R$ and using integration by parts yields

$$
\int \nabla R \cdot \nabla g+\int R g-\int\left(1+\frac{4}{N}\right) R^{\frac{4}{N}+1} g=\int r^{2} R^{\frac{4}{N}+2} .
$$

Combining (3.18) with (3.19), we obtain

$$
\frac{4}{N} \int R^{\frac{4}{N}+1} g=-\int r^{2} R^{\frac{4}{N}+2} .
$$

On the other hand, multiplying (1.7) by $r g^{\prime}(r)$ and integrating over $\mathbf{R}^{N}$ yields

$$
\int\left(R^{\prime \prime}+\frac{N-1}{r} R^{\prime}-R+R^{1+\frac{4}{N}}\right) r g^{\prime}=0 .
$$

Integrating by parts and using (3.14) yields

$$
(N-2) \int \nabla R \cdot \nabla g-\int r R g^{\prime}+\int r R^{1+\frac{4}{N}} g^{\prime}-\int r R^{\prime}\left(g-\left(1+\frac{4}{N}\right) R^{\frac{4}{N}} g-r^{2} R^{\frac{4}{N}+1}\right)=0 .
$$

A direct calculation shows that

$$
(N-2) \int \nabla R \cdot \nabla g+N \int R g-N \int R^{\frac{4}{N}+1} g-\frac{N}{2} \int r^{2} R^{\frac{4}{N}+2}=0 .
$$

Combining (3.19) with (3.21) yields

$$
2 \int R g+2\left(1-\frac{4}{N}\right) \int R^{\frac{4}{N}+1} g=-\frac{N}{2}\left(1-\frac{N}{2}\right) \int r^{2} R^{\frac{4}{N}+2}
$$

Therefore $\int R g=0$ directly follows from (3.20).

By Lemma 3.5, it follows that $\int r^{2} R^{\frac{4}{N}+1} g \geq 0$. Now we claim: $\int r^{2} R^{\frac{4}{N}+1} g>0$, or $\left(-L_{0} g, g\right)>0$. It is observed that

$$
0=\min _{\substack{(f, R)=0 \\ f \in H^{1}}}\left(-L_{0} f, f\right) \leq \min _{\substack{(f, R)=0 \\ f \in H_{r}^{1} \\|f|_{2}=1}}\left(L_{0} f, f\right) \leq\left(-L_{0} v, v\right)
$$

where $v=\frac{g}{|g|_{2}}$.

Therefore, to prove $\left(-L_{0} g, g\right)>0$, it suffices to show the following lemma. 
Lemma 3.6. $\min _{\substack{(f, R)=0 \\ f \in H=\\|f|_{2}=1}}\left(-L_{0} f, f\right)>0$.

Proof of Lemma 3.6. If not, assume that

$$
\min _{\substack{(f, R)=0 \\ f \in H_{r}^{\prime} \\|f|_{2}=1}}\left(-L_{0} f, f\right)=0
$$

Let $\left\{f_{j}\right\}$ be a sequence in $H_{r}^{1}\left(\mathbf{R}^{N}\right)$ with $\left|f_{j}\right|_{2}=1,\left(f_{j}, R\right)=0,\left(-L_{0} f_{j}, f_{j}\right) \geq 0$ and

$$
\lim _{j \rightarrow \infty}\left(-L_{0} f_{j}, f_{j}\right)=0 .
$$

Then, $\forall \delta>0, \exists N_{0}>0$ such that for $j>N_{0}, 0<\left(-L_{0} f_{j}, f_{j}\right)<\delta$ or

$$
1 \leq\left|\nabla f_{j}\right|_{2}^{2}+\left|f_{j}\right|_{2}^{2} \leq\left(1+\frac{4}{N}\right) \int R^{\frac{4}{N}} f_{j}^{2}+\delta .
$$

Since $|R|_{\infty}<\infty,(3.23)$ implies $f_{j}$ is uniformly bounded in $H_{r}^{1}$ for $j \rightarrow \infty$. By standard arguments, it follows that there is a subsequence of the $\left\{f_{j}\right\}$, which is denoted again by $\left\{f_{j}\right\}$, and a $f^{*} \in H_{r}^{1}\left(\mathbf{R}^{N}\right)$ such that $f_{j} \rightarrow f^{*}$ weekly in $H_{r}^{1}\left(\mathbf{R}^{N}\right), f_{j} \rightarrow f^{*}$ a.e. $\mathbf{R}^{N}$, and $f_{j} \rightarrow f^{*}$ a.e. $L_{L o c}^{2}\left(\mathbf{R}^{N}\right)$. On the other hand, we have

$$
\left(f^{*}, R\right)=\lim _{j \rightarrow \infty}\left\langle f_{j}, R\right\rangle=0 .
$$

Because of the exponential decay of $R$ to 0 as $r \rightarrow \infty$ and local convergence of $f_{j}$ in $L_{2}$, we obtain that

$$
\int R^{\frac{4}{N}} f_{j}^{2} \rightarrow \int R^{\frac{4}{N}}\left(f^{*}\right)^{2} \quad j \rightarrow \infty .
$$

Taking the limit in $(3.23)$ yields $1 \leq\left(1+\frac{4}{N}\right) \int R^{\frac{4}{N}}\left|f^{*}\right|^{2}+\delta$. As $\delta>0$, it must be the case that $f^{*} \neq 0$. It is now shown that the infimum is achieved. Indeed,

$$
\left|\nabla f^{*}\right|_{2} \leq \liminf _{j \rightarrow \infty}\left|\nabla f_{j}\right|_{2} \text {. }
$$

Since $\left(R^{\frac{4}{N}} f_{j}, f_{j}\right) \rightarrow\left(R^{\frac{4}{N}} f^{*}, f^{*}\right)$ as $j \rightarrow \infty$, it is deduced that

$$
0 \leq\left(-L_{0} f^{*}, f^{*}\right) \leq \liminf _{j \rightarrow \infty}\left(-L_{0} f_{j}, f_{j}\right)=0 .
$$

Since $f^{*} \neq 0$, define $g^{*}=\frac{f^{*}}{\left|f^{*}\right|_{2}}$. Then we have $g^{*} \in H_{r}^{1},\left|g^{*}\right|_{2}=1,\left(g^{*}, R\right)=0$, and $\left(-L_{0} g^{*}, g^{*}\right)=0$. Consequently, there exist nontrivial critical points $\left(g^{*}, \alpha, \beta\right)$ for the Lagrange multiplier problem,

$$
-L_{0} g^{*}=\alpha g^{*}+\beta R \text { subject to }\left|g^{*}\right|_{2}=1 \text { and }\left(g^{*}, R\right)=0 .
$$

Using (3.24) and the fact that $\left(g^{*}, R\right)=0$, it is easy to see $\alpha=0$. Therefore,

$$
-L_{0} g^{*}=\beta R \text {. }
$$

On the other hand, let $f=\frac{N}{4} R(r)-\frac{1}{2} r R^{\prime}(r)$. Then $-L_{0} f=R$. It follows from (3.25) that $-L_{0}\left(g^{*}-\beta f\right)=0$, that is, $g^{*}-\beta f \in \operatorname{Ker}\left(-L_{0}\right)$. But $\operatorname{Ker}\left(-L_{0}\right)=$ $\operatorname{Span}\left\{R_{x_{i}} \mid i=1,2, \ldots N\right\}$ (see [13]) implies $g^{*}-\beta f$ is not a function of $r$. This is a contradiction with the fact that $g^{*}$ and $f$ are the functions of $r$, i.e., radiallysymmetric functions. Therefore, it is shown that

$$
\min _{\substack{f, R \\\left(f,=0 \\ f f_{2}=1 \\ f \in H_{r}\right.}}\left(-L_{0} f, f\right)>0 .
$$

The proof of Lemma 3.6, as well as Theorem 3.4, is therefore completed. 


\section{INSTABILITY BY BLOW UP}

In this section, we will prove the strong instability of standing waves $\psi=$ $e^{i \omega t} \varphi_{\omega}(r)$ (Theorem 2.5). The method is based on the idea by Cazenave in [2] to construct some invariant sets of the flow of (1.2). Here we construct some crossconstrained invariant sets for the inhomogeneous nonlinearities. Then we apply the virial identity to obtain the blow-up solution for a small perturbation of $\psi=e^{i \omega t} \varphi_{\omega}$.

The following additional assumptions on $V$ are needed in the proof of a strong instability of the standing wave (Theorem 2.5):

$$
\lambda^{2}\left(\frac{p N}{2} V(\varepsilon r)-V^{\prime}(\varepsilon r)(\varepsilon r)-\lambda^{\frac{p N}{2}}\left(\frac{p N}{2} V\left(\frac{\varepsilon r}{\lambda}\right)-V^{\prime}\left(\frac{\varepsilon r}{\lambda}\right) \frac{\varepsilon r}{\lambda}\right)>0\right.
$$

for all $0<\lambda<1$ and $r>0$.

$$
\left(\frac{p N}{2}-2\right) V(\varepsilon r)-V^{\prime}(\varepsilon r) \varepsilon r-\left(\frac{p N}{2}-2\right) \lambda^{\frac{p N}{2}} V\left(\frac{\varepsilon r}{\lambda}\right)+\lambda^{\frac{p N}{2}} V^{\prime}\left(\frac{\varepsilon r}{\lambda}\right) \frac{\varepsilon}{\lambda}>0
$$

for all $0<\lambda<1$ and $r>0$.

Define the set $M$ as

$$
M=\left\{u \in H_{r}^{1}\left(\mathbf{R}^{N}\right) ; \quad P(u)=0, I(u)<0\right\},
$$

where $I(u)$ and $P(u)$ are defined in (2.2) and (2.3), respectively.

Lemma 4.1. (a) Assume $p>4 / N$ or $p=4 / N$ with $V^{(4)}(0)>0$ for a small $\epsilon>0$. Then the assumptions $H_{1}$ and $H_{2}$ hold.

(b) For a small $\epsilon>0$, the set $M$ is nonempty, if

1) $p=4 / N, V^{(4)}(0)>0$ or

2) $p>4 / N, V^{\prime \prime}(0)<0$ or

3) $4 / N<p<8 / N, V^{\prime \prime}(0)=0$ and $V^{(4)}(0)>0$.

Proof of Lemma 4.1. For part (a), first we prove the assumption that $H_{1}$ holds. In fact,

$$
\begin{aligned}
& \lambda^{2}\left(\frac{p N}{2} V(\varepsilon r)-V^{\prime}(\varepsilon r)(\varepsilon r)\right)-\lambda^{\frac{p N}{2}}\left(\frac{p N}{2} V\left(\frac{\varepsilon r}{\lambda}\right)-V^{\prime}\left(\frac{\varepsilon r}{\lambda}\right) \frac{\varepsilon r}{\lambda}\right) \\
= & \lambda^{2}\left[\frac{p N}{2} V(0)+\left(\frac{p N}{4}-1\right) V^{\prime \prime}(0)(\varepsilon r)^{2}+\left(\frac{p N}{2 \cdot 4 !}-\frac{1}{3 !}\right) V^{(4)}(0)(\varepsilon r)^{4}\right. \\
& \left.+O\left((\varepsilon r)^{6}\right)\right]-\lambda^{\frac{p N}{2}}\left[\frac{p N}{2} V(0)+\left(\frac{p N}{4}-1\right) V^{\prime \prime}(0)(\varepsilon r)^{2}\right. \\
+ & \left.\left(\frac{p N}{2 \cdot 4 !}-\frac{1}{3 !}\right) \frac{V^{(4)}(0)}{\lambda^{4}}(\varepsilon r)^{4}+O((\varepsilon r))^{6}\right] \\
= & \left(\lambda^{2}-\lambda^{\frac{p N}{2}}\right) \frac{p N}{2} V(0)+\left(\lambda^{2}-\lambda^{\frac{p N}{2}}-2\right)\left(\frac{p N}{4}-1\right) V^{\prime \prime}(0)(\varepsilon r)^{2} \\
& +\left(\frac{p N}{2 \cdot 4 !}-\frac{1}{3 !}\right)\left(\lambda^{2}-\lambda^{\frac{p N}{2}-4}\right) V^{(4)}(0)\left((\varepsilon r)^{4}\right)+O\left((\varepsilon r)^{6}\right)>0
\end{aligned}
$$

if $p>4 / N$ or $p=4 / N$ and $V^{(4)}(0)>0$ with $\varepsilon \rightarrow 0$. 
For assumption $H_{2}$, we calculate for $0<\lambda<1, \quad r>0$

$$
\begin{aligned}
& \left(\frac{p N}{2}-2\right) V(\varepsilon r)-V^{\prime}(\varepsilon r) \varepsilon r-\left(\frac{p N}{2}-2\right) \lambda^{\frac{p N}{2}} V\left(\frac{\varepsilon r}{\lambda}\right)+\lambda^{\frac{p N}{2}} V^{\prime}\left(\frac{\varepsilon r}{\lambda}\right) \frac{\varepsilon}{\lambda} \\
= & \left(\frac{p N}{2}-2\right)\left[\left(1-\lambda^{\frac{p N}{2}}\right) V(0)+O\left((\varepsilon r)^{2}\right)\right] \\
& -\left(1-\lambda^{\frac{p N}{2}-2}\right) V^{\prime \prime}(0)(\varepsilon r)^{2}-\frac{V^{(4)}(0)}{3 !}(\varepsilon r)^{4}+\frac{\lambda^{\frac{p N}{2}}}{3 !} V^{(4)}(0)\left(\frac{\varepsilon}{\lambda}\right)^{4}+O((\varepsilon r))^{6} \\
= & \left(\frac{p N}{2}-2\right)\left(1-\lambda^{\frac{p N}{2}}\right) V(0)+\left(\frac{p N}{2}-2\right) O\left((\varepsilon r)^{2}\right) \\
& -\left(1-\lambda^{\frac{p N}{2}-2}\right) V^{\prime \prime}(0)(\varepsilon r)^{2}-\frac{1}{3 !} V^{(4)}(0)\left(1-\lambda^{\frac{p N}{2}-4}\right)(\varepsilon r)^{4}+O\left((\varepsilon r)^{6}\right)>0
\end{aligned}
$$

if $p>4 / N$ or $p=4 / N$ and $V^{(4)}(0)>0$ with $\varepsilon \rightarrow 0$.

To finish the proof of part (b) of Lemma 4.1, we define $\varphi_{\lambda}=\lambda \varphi(x)$ with $\lambda>0$ where $\varphi_{1}=\varphi$ is the ground state of

$$
\triangle \varphi-\omega \varphi+V(0) \varphi^{p+1}=0 .
$$

A simple calculation shows that

$$
\begin{aligned}
& \int|\nabla \varphi|^{2}+\omega \int|\varphi|^{2}-\int V(0)|\varphi|^{p+2}=0 \text { and } \\
& \frac{1}{2} \int|\nabla \varphi|^{2}-\frac{1}{2(p+2)} \int \frac{p N}{2} V(0)|\varphi|^{p+2}=0 .
\end{aligned}
$$

For a small $\varepsilon>0$, we estimate

$$
P\left(\varphi_{\lambda}\right)=\frac{1}{2} \lambda^{2} \int|\nabla \varphi|^{2}-\frac{\lambda^{p+2}}{2(p+2)} \int\left(\frac{p N}{2} V(\varepsilon r)-V^{\prime}(\varepsilon r)(\varepsilon r)\right)|\varphi|^{p+2}
$$

and

$$
\begin{aligned}
P(\varphi)= & \frac{1}{2}|\nabla \varphi|^{2}-\frac{1}{2(p+2)} \int\left[\frac{p N}{2} V(0)+\frac{p N}{4} V^{\prime \prime}(0)(\varepsilon r)^{2}\right. \\
& \left.-V^{\prime \prime}(0)(\varepsilon r)^{2}+\frac{p N}{2 \cdot 4 !} V^{(4)}(0)(\varepsilon r)^{4}-\frac{1}{3 !} V^{(4)}(0)(\varepsilon r)^{4}+O\left((\varepsilon r)^{6}\right)\right]|\varphi|^{p+2} \\
= & \frac{1}{2} \int|\nabla \varphi|^{2}-\frac{1}{2(p+2)} \int \frac{p N}{2} V(0)|\varphi|^{p+2} \\
& -\frac{1}{2(p+2)}\left(\frac{p N}{4}-1\right) V^{\prime \prime}(0) \int(\varepsilon r)^{2}|\varphi|^{p+2} \\
& -\left(\frac{p N}{8}-1\right) \frac{V^{(4)}(0)}{4 !\left(\frac{p}{2}+1\right)} \int\left((\varepsilon r)^{4}+O\left((\varepsilon r)^{6}\right)\right)|\varphi|^{p+2} \\
= & -\frac{1}{2(p+2)}\left(\frac{p N}{4}-1\right) V^{\prime \prime}(0) \int(\varepsilon r)^{2}|\varphi|^{p+2} \\
& -\left(\frac{p N}{8}-1\right) \frac{V^{(4)}(0)}{4 !\left(\frac{p}{2}+1\right)} \int\left[(\varepsilon r)^{4}+O\left((\varepsilon r)^{6}\right)\right]|\varphi|^{p+2} .
\end{aligned}
$$

Since $\varphi$ is exponentially decay at $r, P(\varphi)>0$ under the conditions (a) or (b). On the other hand, for a small $\varepsilon>0$ and $\lambda \rightarrow \infty$

$$
P\left(\varphi_{\lambda}\right)=\frac{\lambda^{2}}{2} \int|\nabla \varphi|^{2}-\frac{\lambda^{p+2}}{2(p+2)} \int\left(\frac{p N}{2} V(0)+O\left((\varepsilon r)^{2}\right)\right)|\varphi|^{p+2}<0 .
$$


By continuity of $P\left(\varphi_{\lambda}\right)$ for $\lambda \in[1, \infty)$, there exists $\mu \in(1, \infty)$ such that $P\left(\varphi_{\mu}\right)=0$. To prove $I\left(\varphi_{\mu}\right)<0$, we estimate that

$$
\begin{aligned}
I\left(\varphi_{\mu}\right)= & \mu^{2} \omega \int|\varphi|^{2}+\mu^{2} \int|\nabla \varphi|^{2}-\mu^{p+2} \int V(\varepsilon)|\varphi|^{p+2} \\
= & \mu^{2} \omega \int|\varphi|^{2}+\mu^{2} \int|\nabla \varphi|^{2}-\mu^{p+2} \int\left(V(0)+O\left((\varepsilon r)^{2}\right)\right)|\varphi|^{p+2} \\
= & \mu^{2}\left[\omega \int|\varphi|^{2}+\int|\nabla \varphi|^{2}-\int V(0)|\varphi|^{p+2}\right] \\
& -\left(\mu^{p+2}-\mu^{2}\right) \int\left(V(0)+O\left((\varepsilon r)^{2}\right)\right)|\varphi|^{p+2} \\
= & -\mu^{2}\left(\mu^{p}-1\right) \int(V(0)+O((\varepsilon r)))|\varphi|^{p+2}<0 .
\end{aligned}
$$

Since $\mu>1$, this implies $\varphi_{\mu} \in M$. The proof of Lemma 4.1 is complete.

The proof of the strong instability result (Theorem 2.5) is approached via a series lemmas. Define $d_{M}$ as follows:

$$
d_{M}=\inf \{L(v) ; \quad v \neq 0, v \in M\} .
$$

Lemma 4.2. Under the same assumptions of $V, p$ and $N$ in Lemma $4.1, d_{M} \geq$ $d(\omega)$, where $d(\omega)$ is defined in (1.8).

Proof. Let $u^{\lambda}=\lambda^{N / 2} u(\lambda x)$, for $u \in M$. Then one has

$$
\begin{gathered}
I\left(u^{\lambda}\right)=\omega \int|u|^{2}+\lambda^{2} \int|\nabla u|^{2}-\lambda^{\frac{p N}{2}} \int V\left(\frac{\epsilon r}{\lambda}\right)|u|^{p+2}, \\
P\left(u^{\lambda}\right)=\lambda^{2} \int|\nabla u|^{2}-\frac{\lambda^{\frac{p N}{2}}}{p+2} \int\left(\frac{p N}{2} V\left(\frac{\epsilon r}{\lambda}\right)-V^{\prime}\left(\frac{\epsilon r}{\lambda}\right) \frac{\epsilon r}{\lambda}\right)|u|^{p+2}, \\
L\left(u^{\lambda}\right)=\frac{1}{2} \lambda^{2} \int|\nabla u|^{2}-\frac{1}{p+2} \lambda^{\frac{p N}{2}} \int V\left(\frac{\epsilon r}{\lambda}\right)|u|^{p+2}+\frac{\omega}{2} \int|u|^{2},
\end{gathered}
$$

and

$$
\frac{\partial}{\partial \lambda} L\left(u^{\lambda}\right)=\frac{1}{\lambda} P\left(u^{\lambda}\right)
$$

Since $u \in M$, there exists $\mu \in(0,1)$ such that $I\left(u^{\mu}\right)=0$. Indeed, at $\lambda \rightarrow 0, I\left(u_{0}\right) \rightarrow$ $\int|u|^{2}>0$, and $I\left(u^{\lambda}\right)<0$ at $\lambda=1$ by the definition of $M$. Applying the relation $P(u)=0$ and using (4.8), one obtains

$$
\begin{aligned}
\lambda \frac{\partial}{\partial \lambda} L\left(u^{\lambda}\right) & =\frac{\lambda^{2}}{p+2} \int\left(\frac{p N}{2} V(\epsilon r)-V^{\prime}(\epsilon r)(\epsilon r)\right)|u|^{p+2} \\
& -\frac{\lambda^{\frac{p N}{2}}}{p+2} \int\left(\frac{p N}{2} V\left(\frac{\epsilon r}{\lambda}\right)-V^{\prime}\left(\frac{\epsilon r}{\lambda}\right) \frac{\epsilon r}{\lambda}\right)|u|^{p+2} .
\end{aligned}
$$

Under the conditions of Lemma 4.1, $V$ satisfies the assumption $H_{1}$. Therefore $\frac{\partial}{\partial \lambda} L\left(u^{\lambda}\right)>0$ when $\lambda \in(\mu, 1)$, and $L\left(u^{\lambda}\right)$ reaches the minimal at $\mu$. Since $I\left(u^{\mu}\right)=0$, we have $L\left(u^{\mu}\right) \geq d(\omega)$ as the definition of $d(\omega)$. Therefore $L(u) \geq L\left(u^{\mu}\right) \geq d(\omega)$, which implies that $d_{M} \geq d(\omega)$. This completes the proof of Lemma 4.2.

Define a set $\Gamma_{\omega}$ as

$$
\Gamma_{\omega}=\left\{u \in H_{r}^{1}\left(\mathbf{R}^{N}\right) ; \quad L(u)<d(\omega), P(u)<0, I(u)<0\right\},
$$

where $L, P, I$ and $d(\omega)$ are defined in (2.1), (2.3), (2.2) and (1.8), respectively. 
Lemma 4.3. Under the same conditions of $V, p$ and $N$ in Lemma 4.1, $\Gamma_{\omega}$ is invariant under the flow of (1.2) in the sense that: If $u_{0} \in \Gamma_{\omega}$, then the unique solution $u(t)$ of (1.2), $0 \leq t<T$, with the initial data $u_{0}$ satisfies

$$
u(t) \in \Gamma_{\omega}, \quad \text { for } t \in[0, T),
$$

where $T>0$ is the maximum existence time of the solution $u(t)$.

Proof. It is observed by the conservation laws that $L(u(t))=L\left(u_{0}\right)<d(\omega)$, for $0 \leq t<T$. If there exists $t_{0}>0$ such that $I\left(u\left(t_{0}\right)\right)=0$, then by Lemma 2.2, we get $L\left(u\left(t_{0}\right)\right) \geq d(\omega)$, and this shows that $d(\omega) \leq L\left(u\left(t_{0}\right)\right)<d(\omega)$. This is a contradiction. Therefore

$$
I(u(t))<0, \quad \text { for } t \in[0, T) .
$$

If there exists $t_{1}>0$ such that $P\left(u\left(t_{1}\right)\right)=0$, then $u\left(t_{1}\right) \in M$ by (4.13). It follows that $L\left(u\left(t_{1}\right)\right) \geq d_{M} \geq d(\omega)$, which is a contradiction. Therefore the proof of Lemma 4.3 is complete.

Lemma 4.4. Under the assumptions of Lemma 4.1, the solution $u(t)$ of (1.2) with the initial data $u_{0} \in \Gamma_{\omega}$ blows up in finite time.

Proof. Let $u_{0} \in \Gamma_{\omega}$. Since $I\left(u_{0}\right)<0$, following the same arguments as in Lemma 4.2, there exists $\mu \in(0,1)$ such that $I\left(u_{0}^{\mu}\right)=0$ and $I\left(u_{0}^{\lambda}\right)<0$ for any $\lambda \in(\mu, 1)$, where $u_{0}^{\lambda}=\lambda^{N / 2} u_{0}(\lambda x)$. A direct calculation shows that for any $\eta \in(0,1)$, we have

$L\left(u_{0}\right)-L\left(u_{0}^{\eta}\right)=\frac{1}{2} P\left(u_{0}\right)-\frac{1}{2} P\left(u_{0}^{\eta}\right)$

$$
\begin{aligned}
& +\frac{1}{2(p+2)} \int\left(\left(\frac{p N}{2}-2\right) V(\epsilon r)-V^{\prime}(\epsilon r)(\epsilon r)\right)\left|u_{0}\right|^{p+2} \\
& -\frac{1}{2(p+2)} \int\left(\left(\frac{p N}{2}-2\right) \eta^{\frac{p N}{2}} V\left(\frac{\epsilon r}{\eta}\right)-\eta^{\frac{p N}{2}} V^{\prime}\left(\frac{\epsilon r}{\eta}\right) \frac{\epsilon r}{\eta}\right)\left|u_{0}\right|^{p+2} .
\end{aligned}
$$

Since the assumption $\mathrm{H}_{2}$ is satisfied, it turns out that

$$
L\left(u_{0}\right)-L\left(u_{0}^{\eta}\right)>\frac{1}{2} P\left(u_{0}\right)-\frac{1}{2} P\left(u_{0}^{\eta}\right) .
$$

Since $P\left(u_{0}\right)<0$, we have two possibilities:

i) $P\left(u_{0}^{\mu}\right) \leq 0$ or

ii) there exists some $\xi \in(\mu, 1)$ such that $P\left(u_{0}^{\xi}\right)=0$ and $I\left(u_{0}^{\xi}\right)<0$.

In case i), we have

$$
L\left(u_{0}\right)-L\left(u_{0}^{\mu}\right)>\frac{1}{2} P\left(u_{0}\right)-\frac{1}{2} P\left(u_{0}^{\mu}\right) \geq \frac{1}{2} P\left(u_{0}\right) .
$$

While in case ii),

$$
L\left(u_{0}\right)-L\left(u_{0}^{\xi}\right) \geq \frac{1}{2} P\left(u_{0}\right)-\frac{1}{2} P\left(u_{0}^{\xi}\right)=\frac{1}{2} P\left(u_{0}\right) .
$$

Clearly $I\left(u_{0}^{\mu}\right)=0$ and $u_{0}^{\xi} \in M$ in case i) and ii), respectively. So we have $L\left(u_{0}^{\mu}\right) \geq$ $d(\omega)$ or $L\left(u_{0}^{\xi}\right) \geq d_{M} \geq d(\omega)$ by Lemma 4.2. Consequently, $P\left(u_{0}\right)<2\left(L\left(u_{0}\right)-\right.$ $d(\omega))=-\delta<0$, for any $u_{0} \in \Gamma_{\omega}$, where $\delta$ is a fixed positive number determined by $u_{0}$. Applying the virial identity $(2.5)$

$$
\frac{d^{2}}{d t^{2}} \int r^{2}|u(r, t)|^{2}=8 P(u(t))
$$


and the invariant sets of $\Gamma_{\omega}$, it follows from the inequality $|u|_{2} \leq \frac{2}{N}|\nabla u|_{2}|r u|_{2}$ that the solution $u(t)$ of (1.2) with the initial data $u_{0} \in \Gamma_{\omega}$ blows up in finite time. This completes the proof of Lemma 4.4 .

By Lemma 4.4, we are able to prove Theorem 2.5.

Proof of Theorem 2.5. Let $\varphi_{\omega}$ be the ground state of (1.3). Then we have the Pohozaev's identities $I\left(\varphi_{\omega}\right)=P\left(\varphi_{\omega}\right)=0$. Let $\varphi_{\omega}^{\lambda}=\lambda^{N / 2} \varphi_{\omega}(\lambda x)$ with $\lambda>1$. From (4.9) and (4.10) and the assumption $H_{1}$, it follows that

$$
\frac{d}{d \lambda} L\left(\varphi_{\omega}^{\lambda}\right)=\frac{1}{\lambda} P\left(\varphi_{\omega}^{\lambda}\right)<0
$$

when $\lambda>1$. Note that the assumption $H_{1}$ changes the sign to negative if $\lambda>1$. Moreover, since $P\left(\varphi_{\omega}\right)=0$,

$$
\left.\frac{d}{d \lambda} I\left(\varphi_{\omega}^{\lambda}\right)\right|_{\lambda=1}=-p \int\left|\nabla \varphi_{\omega}\right|^{2}<0 .
$$

Therefore, as $\lambda \rightarrow 1^{+}, L\left(\varphi_{\omega}^{\lambda}\right)<d(\omega), P\left(\varphi_{\omega}^{\lambda}\right)<0$ and $I\left(\varphi_{\omega}^{\lambda}\right)<0$, i.e. $\varphi_{\omega}^{\lambda} \in \Gamma_{\omega}$. It follows from Lemma 4.4 that the solution $u$ of (1.2) with such an initial data $u(0)=\varphi_{\omega}^{\lambda}$ blows up in finite time. On the other hand, it is clear that $\varphi_{\omega}^{\lambda} \rightarrow \varphi_{\omega}$ in $H_{r}^{1}\left(\mathbf{R}^{N}\right)$ as $\lambda \rightarrow 1^{+}$. This completes the proof of Theorem 2.5.

\section{ACKNOWLEDGMENTS}

The authors thank the referee for the careful reading of the manuscript and the valuable comments. This work is supported in part by RGC Competitive Earmarked Research Grant HKUST 6176/99P and HKUST 6143/01P.

\section{REFERENCES}

[1] Berestycki, $\mathrm{H}$ and Cazenave, T., Instabilityé des états stationnaires dans les équations de Schrödinger et de Klein-Gordon non linéaires, C. R. Acad Sc. Paris, 293 (1981), 489-492. MR0646873 (84f:35120)

[2] Cazenave, T., An introduction to nonlinear Schrödinger equation, Textos de Métodos Matemaáticos 26 (1989), Instituto de Matemática, UFRJ, Rio de-Janeiro.

[3] Cazenave, T. and Lions, P. L., Orbital stability of standing waves for some nonlinear Schrödinger equations, Comm. Math. Phys., 85 (1982), 549-561. MR0677997 (84i:81015)

[4] Fibich, G. and Wang, X. P., Stability of solitary waves for nonlinear Schrödinger equations with inhomogeneous nonlinearities, Physica D ; 175, (2003) P96-108. MR1957907 (2003m:35225)

[5] Gill, T. S., Optical guiding of laser beam in nonuniform plasma, Pramana Journal of Physics, 55 (2000), 845-852.

[6] Ginibre J. and Velo, G., On a class of nonlinear Schrödinger equations I, II. The Cauchy problem, general case, J. Func. Anal., 32 (1979), 1-71. MR0533218(82c:35057) MR0533219 (82c:35058)

[7] Goncalves Rebeiro, J. M., Instability of symmetric stationary states for some nonlinear Schrödinger equations with an external magnetic field, Ann. Inst. Henri Poincaré Physique Théorique, 54 (1991), 403-433. MR1128864 (92i:35113)

[8] Grillakis, M., Shatah, J. and Strauss, W., Stability theory of solitary waves in the presence of symmetry, I, J. Funct. Anal. 74 (1987), 160-197. MR0901236 (88g:35169)

[9] Kato, T., On the blowing-up of solutions, Ann. Inst. Henri Poincaré, Physique Théorique, 49 (1987), 113-129.

[10] Liu, Yue and Wang, X. P., Nonlinear stability of solitary waves of a generalized KadomtsevPetvishvili equation, Comm. Math. Phys. 183 (1997), 253-266. MR1461958 (99b:35184) 
[11] Merle, F., Nonexistence of minimal blow-up solutions of equations iu $u_{t}=\Delta u-k(x)|u|^{4 / N} u$ in $R^{N}$, Ann. Inst. Henri Poincaré Physique Théorique, 64 (1996), 33-85. MR.1378233 (97g:35073)

[12] Shatah, J. and Strauss, W., Instability of nonlinear bound states, Comm. Math. Phys., 100 (1985), 173-190. MR0804458 (87b:35159)

[13] Weinstein, M. I., Modulational stability of ground states of nonlinear Schrödinger equations, SIAM J. Math. Anal., 16 (1985), 472-491. MR0783974 (86i:35130)

[14] Weinstein, M. I., Nonlinear Schrödinger equations and sharp interpolation estimates, Comm. Math. Phys., 87 (1983), 567-576. MR0691044 (84d:35140)

[15] Wang, K., On the strong instability of standing wave solutions of inhomogeneous NLS equation, Thesis, Department of Mathematics, The Hong Kong University of Science and Technology, 2001.

[16] Wang, X. F. and Zeng, B., On concentration of positive bound states of nonlinear Schrödinger equations with competing potential functions, SIAM J. Math. Anal. 28 (1997), 633-655. MR $1443612(98 \mathrm{e}: 81032)$

Department of Mathematics, University of Texas, Arlington, Texas 76019

E-mail address: yliu@uta.edu

Department of Mathematics, The Hong Kong University of Science and Technology, Clear Water Bay, Kowloon, Hong Kong

E-mail address: mawang@ust.hk

California Institute of Technology, MC 217-50, 1200 E. California Boulevard, Pasadena, California 91125

E-mail address: wang@acm.caltech.edu 